\title{
Inaugural Issue Editorial for ACM Transactions on Quantum Computing
}

Welcome to the inaugural issue of ACM Transactions on Quantum Computing (TQC), a high-impact, peer-reviewed journal that supports the quantum computing community. As the editors-in-chief for TQC, we are delighted to introduce this new journal at a time when quantum computing, and quantum information broadly, are rapidly developing.

In recent years, research groups worldwide, as well as both large and small companies, have begun developing quantum computers and computing devices that go beyond the prototype stage toward useful computational tools. The computer science community has already started to understand, evaluate, and build infrastructure around this novel paradigm. Our journal, TQC, is dedicated to providing a central venue for research in this new territory of computer science.

The journal aims to publish high-quality papers on both the theory and practice of quantum computing, including but not limited to models of quantum computing, quantum algorithms and complexity, quantum computing architecture, principles and methods of fault-tolerant quantum computation, design automation for quantum computing, issues surrounding compilers for quantum hardware and noisy intermediate-scale quantum implementations, quantum programming languages and systems, distributed quantum computing, quantum networking, quantum security and privacy, and applications (e.g., machine learning and AI) of quantum computing.

Quantum computing is interdisciplinary by nature, and the field has been developed from its beginning through the combined effort of researchers from different areas, including computer science, physics, engineering, and mathematics, among many others. We particularly welcome submissions reporting innovative research at the intersection of computer science and these other areas, as well as the application of quantum computing technologies to domains such as quantum simulation, machine learning, and mathematics.

We wish to thank the many people who have helped to envision and establish TQC as a highcaliber journal for the quantum community. We are grateful to the members of advisory board for their strong support. We would like to thank our esteemed editorial board members who have joined with enthusiasm in participating in founding this journal. And, of course, we are grateful to the ACM for providing a home for the emerging innovations of quantum computer science.

This first issue of TQC presents a collection of five outstanding research papers that capture the breadth and sophistication of quantum computing research. Baker et al. (https://doi.org/10. 1145/3406309) propose a novel technique for decomposition of a large class of quantum circuits that can achieve a significant improvement of depth over the best-known qubit-only techniques. Flammia and Wallman (https://doi.org/10.1145/3408039) present an efficient procedure for characterizing Pauli channels, which are an important noise model in many practical quantum computing architectures. as well as the analysis of error correction and fault tolerance technology.

\footnotetext{
ACM Reference format:

https://doi.org/10.1145/3411487

(c) 2020 Copyright held by the owner/author(s).

2643-6817/2020/12-ART1

https://doi.org/10.1145/3411487
}

Travis S. Humble and Ying Mingsheng. 2020. Inaugural Issue Editorial for ACM Transactions on Quantum Computing. ACM Trans. Quantum Comput. 1, 1, Article 1 (December 2020), 2 pages. 
Das and Paul (https://doi.org/10.1145/3396239) demonstrate new methods in error modeling tailored to experimental tests of Hardy's paradox in quantum foundations using non-locality on a superconducting quantum computer. Kerenidis and Prakash (https://doi.org/10.1145/3406306) propose a quantum interior point method for solving two fundamental problems in optimizationlinear programming and semi-definite programming-that offers significant speedup over the bestknown classical solvers with potential applications in machine learning. Allcock et al. (https:// doi.org/10.1145/3411466) develop quantum machine learning algorithms for training and evaluating feedforward neural networks that can be quadratically faster in the size of the network than their classical counterparts.

\author{
Travis S. Humble \\ Mingsheng Ying \\ Co-Editors-in-Chief
}

